\title{
La salud general percibida y su asociación al síndrome de burnout académico e ideación suicida en universitarios colombianos
}

\section{Perceived general health and its association with academic burnout syndrome and suicide ideation in Colombian university students}

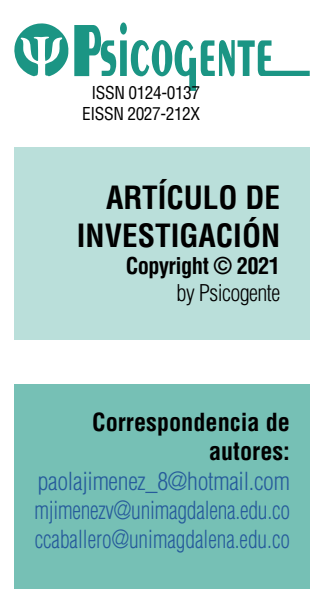

Recibido: $10-02-2020$ Aceptado: $10-08-2020$ Publicado: 01-01-2021
María Paola Jiménez Villamizar (iD - Carmen Cecilia Caballero Domínguez Universidad del Magdalena, Santa Marta, Colombia

Resumen

Objetivo: Describir la prevalencia de la salud general percibida y su asociación al síndrome de burnout académico e ideación suicida en universitarios de una institución pública de la ciudad de Santa Marta.

Método: Se realizó un estudio observacional de corte transversal con componente analítico, en una muestra de 807 estudiantes de una universidad pública, quienes respondieron la escala de salud general (GHQ), el inventario de burnout académico (MBI-SS) y la escala de ideación suicida (CES-D-IS).

Resultados: Mostraron el $60 \%$ de los participantes eran mujeres con edades entre 18 a 47 años. El $28 \%$ informa malestar en su salud general percibida. La ideación suicida (OR = 3,16; IC $95 \% 1,82$ - 5,49), el agotamiento (OR = 2,22; IC $95 \% 1,51$ - 3,25), el cinismo (OR = 1,61; IC $95 \% 1,14-2,28)$, y la ineficacia académica (OR = 1,60; IC $95 \%$ 1,12-2,28) se asociaron a malestar general percibido.

Conclusiones: La ideación suicida y las dimensiones del burnout (agotamiento, cinismo e ineficacia académica) son factores de riesgo para la salud general percibida en los estudiantes universitarios. Se necesita más investigación acerca del papel de variables contextuales en la salud general de los estudiantes universitarios.

Palabras clave: salud general percibida; burnout; agotamiento; cinismo; ineficacia académica; ideación suicida.

Abstract

Objective: To describe the prevalence of perceived general health and its association to academic burnout syndrome and suicide ideation in university students of a public institution in the city of Santa Marta.

Method: A cross-sectional observational study with analytical component was conducted in a sample of 807 students from a public university, who answered the general health scale (GHQ), the academic burnout inventory (MBI-SS) and the suicide ideation scale (CES-D-IS).

Results: They showed $60 \%$ of the participants were women between the ages of 18 and $47,28 \%$ reported an impairment in their perceived general health. Suicidal ideation (OR = 3,16; IC $95 \% 1$,82 5,49), exhaustion (OR = 2,22; IC $95 \% 1,51-3,25)$, cynicism (OR = 1,61; IC $95 \% 1,14-2,28)$, and academic inefficacy $(O R=1,60$; IC $95 \%$ 1,12- 2,28) were associated with perceived general malaise.

Conclusions: Suicidal ideation, and burnout dimensions (exhaustion, cynicism and academic inefficiency) are risk factors for perceived general health in university students. More research is needed on the role of contextual variables in the general health of university students.

Key words: perceived general health; burnout; exhaustion; cynicism; academic inefficiency; suicidal ideation. 


\section{INTRODUCCIÓN}

La salud como la ausencia de malestar físico, ha evolucionado en las últimas décadas gracias a los avances en investigaciones desde la perspectiva de la psicología positiva, demostrando el vínculo de la salud con factores positivos y del buen funcionamiento, que van más allá de lo meramente biológico, en el que juega un papel determinante factores psicológicos, culturales y sociales (Olarte, 2018). La salud más que un estado, es considerado como un proceso en el que el sujeto se mueve entre dos polos, malestar y bienestar físico, mental y social, y no se reduce a la ausencia de enfermedades (Organización Mundial de la Salud, 2006). Desde esta perspectiva, la salud abarca, entre otros aspectos, el bienestar subjetivo, la percepción de la propia eficacia, la autonomía, la competencia, la dependencia generacional y la autorrealización de las capacidades intelectuales y emocionales (Organización Mundial de la Salud, 2001).

La Salud General Percibida es el valor subjetivo que le atribuye el individuo a su salud física o mental, expresa el sentir y pensar positivo acerca de sí mismo, en relación con el funcionamiento biopsicosocial: físico, psíquico y social (Victoria y González, 2000; Schwartzmann, 2003). El individuo muchas veces puede sentirse sano y no estarlo, o en su defecto sentirse enfermo y pensar que está sano (Ardila, 2003). El estado de salud se relaciona significativamente con el bienestar psicológico (Seligman \& Peterson, 2003). La capacidad de los individuos para hacer frente al estrés normal de la vida, trabajar productivamente, y contribuir a la comunidad, se logra, entre otras variables, a través de la percepción de bienestar general (Organización Mundial de la Salud, 2004).

Los universitarios en gran medida son adolescentes en transición a la etapa adulta y la manera como perciban su salud puede influir en su desarrollo académico, considerándose como un predictor del desempeño académico (Vaez, Kristenson \& Laflamme, 2004), de la deserción (Borquéz, 2005), de la salud física y mental del estudiante (Vélez \& Roa, 2005). Diversos estudios señalan que la salud en universitarios latinoamericanos se caracteriza por la percepción positiva como "buena o muy buena" (Consorcio de Universidades, 2006; Chau \& Saravia, 2014).

\subsection{Burnout académico y salud general percibida}

Algunos estudiantes logran desarrollar estrategias que le permiten afrontar y resolver las respuestas emocionales de tensión y malestar, mientras que otros presentan dificultades en el proceso de adaptación ante las exigencias 
académicas y los estresores vitales, por lo tanto desarrollan manifestaciones de burnout académico (Guarino, Gavidia, Antor \& Caballero, 2000; Aranceli, Perea \& Ormeño, 2006; Wilcox, Arria, Caldeira, Vincent, Pinchevsky \& O'Grady, 2010; Chau \& Tavera, 2012; Palacio, Caballero, González, Gravini \& Contreras, 2012; Becerra, 2013; Flores, Chávez \& Aragón, 2016).

El burnout académico es un estrés crónico, negativo e insidioso relacionado con el contexto de la organización universitaria y con la experiencia del estudiante frente a los estudios (Caballero, 2012), manifestado por tres dimensiones: agotamiento emocional considerado como, la sensación de sentirse agotado física y emocionalmente, tensión durante la jornada académica y sentirse exhaustos; cinismo, actitud de autocrítica, distanciamiento y pérdida de interés en las tareas académicas e incluso a la carrera; e ineficacia académica, valoración del estudiante que no cuenta con recursos personales para resolver los problemas relacionados con sus estudios de manera eficaz (Salanova, Martínez, Bresó, Llorens \& Grau, 2005; Caballero, Abello \& Palacio, 2007).

El síndrome de burnout académico se caracteriza por un conjunto de síntomas emocionales cognitivos, psicosomáticos y sociales (Carlin \& Garcés de los Fayos, 2010; Rosales \& Rosales, 2013; Balsera et al, 2016). Todos estos síntomas son productos de los desafíos y exigencias que enfrentan los estudiantes afectando su bienestar físico y psicológico (Espinás, Riba, Borrás \& Sánchez, 1995; Gracia, Herrero \& Musitu, 1996; Aranceli, Perea \& Ormeño, 2006; Marenco, Suárez \& Palacio, 2017) y la salud general percibida (Viñas \& Chaparrós, 2000). En ese mismo sentido, Hernández, Marques, Jiménez \& Rodríguez (2016) hallaron en estudiantes universitarios asociación significativa entre cinismo con percepción negativa frente a la salud. Estudios precedentes señalan que entre más alta la eficacia académica mayor es la salud general percibida en universitarios. Desde esta perspectiva, la eficacia en altos niveles, produce efectos positivos en la salud percibida (Bandura, 1997; Liébana, 2012; Schönfeld, Brailovskaia, Bieda, Chi Zhang, \& Margraf, 2016; Martos, Pérez-Fuentes, Del Mar, Gázquez, Del Mar \& Belén, 2018).

\subsection{Ideación suicida y salud general percibida}

La ideación es una de las etapas previas al suicidio consumado, referido al pensamiento o fantasías alrededor de la propia muerte, el individuo se siente sin valor y desea la muerte, es el deseo o plan acerca de cometer un suicidio (Muñoz, Pinto, Callata, Napa \& Perales, 2006). En el contexto universitario se ha encontrado que los estudiantes presentan pensamientos suicidas 
recurrentes a lo largo de su período académico (Arango, Castaño, Henao, Jiménez, López \& Páez, 2010; Aradilla \& Tomás, 2011; Dasgupta \& Hazra, 2011; Ceballos \& Suárez, 2012).

La ideación suicida es un indicador de salud mental, asociado a diversos problemas mentales, que actúan como mediadores en la salud general percibida. Estudios precedentes, han registrado relación entre ideación suicida y percepción negativa de la salud en universitarios (Guarino, Gavidia, Antor \& Caballero, 2000; Calvo, Sánchez \& Tejeda, 2003; Hernández \& Gómez, 2006; Cova, Alvial, Aro, Bonifettu, Hérnandez \& Rodríguez, 2007; Kumar, Dixit, Chaudhury \& Kenswar, 2013). En el malestar psicológico se asoció a ideación suicida en estudiantes de Veracruz (Aburto, Díaz \& López, 2017).

En Colombia, se han adelantado investigaciones en universitarios que permiten conocer comportamientos de riesgo y estilos de vida que pueden afectar su percepción en salud (Herazo, Núñez, Sánchez, Vásquez, Lozano, Torres y Valdelamar, 2020; Hanna, Ocampo, Janna, Mena y Torreglosa, 2020). Sin embargo, la revisión literaria sobre la percepción de salud, su asociación a burnout e ideación suicida con universitarios colombianos, especialmente de la región Caribe, es escasa. El presente estudio, responde a las necesidades actuales de la poca evidencia científica de factores de riesgo a la percepción de salud general en el contexto de la región Caribe de Colombia; y aporta a la comprensión de algunos factores asociados, desde una aproximación de la salud integral, como un proceso de salud y enfermedad de las personas, dinámico y complejo, y además sirve de base para futuras investigaciones. Esta investigación contribuirá a la detección precoz de síntomas nocivos para el bienestar y a la comprensión de las variables relacionadas a la salud general de los estudiantes universitarios. Además, proporciona información para el diseño de programas de prevención selectiva e indicada, adaptada culturalmente al contexto universitario, consolidando a las Instituciones de Educación Superior como organizaciones saludables.

El objetivo del presente estudio es describir la prevalencia de la salud general percibida y su asociación al síndrome de burnout e ideación suicida en universitarios.

\section{MÉTODO}

\subsection{Diseño}

El presente estudio es observacional, analítico y transversal, dado que indaga las variables como el síndrome de burnout e ideación suicida y su asociación 
con la salud general percibida en un solo momento (Hernández-Ávila, Garrido-Latorre \& López-Moreno, 2000).

\subsection{Participantes}

Se contó con una población de 2.890 estudiantes de los programas académicos de las Facultades de Ciencias de la Salud, Humanidades y Ciencias Empresariales de una institución universitaria, se calculó un muestreo multietápico. Inicialmente, se efectuó un muestreo aleatorio simple con un nivel de confianza del $95 \%$ y un margen de error $4 \%$; posteriormente, se seleccionaron los cursos con más de cuatro créditos de manera probabilística y estratificada, los salones de clases se tomaron como conglomerados de 30 estudiantes aproximadamente. La muestra estuvo conformada por 807 estudiantes universitarios, como criterios de inclusión se tuvo en cuenta: estudiantes matriculados en las facultades seleccionadas y ser mayor de 18 años.

\subsection{Instrumentos}

\subsubsection{La evaluación de la Salud General Percibida se llevó a cabo} mediante la Escala de Salud General - GHQ (Villa, Zuluaga \& Restrepo, 2013)

Este instrumento evalúa la dimensión general de la salud autopercibida por el estudiante. Consta de 12 ítems, con una escala tipo Likert de 4 puntos ( 0 a 3), que puede ser transformada en una puntuación dicotómica, llamada puntuación GHQ. La escala está compuesta por dos factores denominados bienestar y malestar percibido. Ejemplo de ítems: ¿Ha sentido que está jugando un papel útil en la vida? El coeficiente de consistencia interna para la escala total es de $(\alpha=0,77)$.

\subsubsection{El Burnout Académico se evalúo con Inventory-Student Survey (MBI-SS) (Schaufeli, et al., 2002)}

Fue adaptado a la población de estudiantes universitarios colombianos por Hederich-Martínez \& Caballero-Domínguez (2016). Este instrumento consta de 14 ítems que evalúan las tres dimensiones que categorizan al síndrome de burnout académico: el agotamiento emocional ( 5 ítems); Cinismo (4 ítems) y Eficacia (5 ítems). En un estudio previo, esta escala mostró un alfa de Cronbach de 0,77 para la dimensión de agotamiento, 0,73 para cinismo y 0,82 ineficacia (Hederich-Martínez \& Caballero-Domínguez, 2016). En el presente estudio el $\alpha$ de Cronbach encontrado para las dimensiones de agotamiento $(0,79)$, cinismo $(0,75)$, eficacia $(0,79)$. Ejemplo de los ítems: Estoy cansado 
cuando me levanto por la mañana y tengo que afrontar otro día en la universidad (agotamiento); Me he distanciado de mis estudios porque pienso que no serán realmente útiles (cinismo); Puedo resolver de manera eficaz los problemas relacionados con mis estudios (eficacia).

\subsubsection{La ideación Suicida se evaluó mediante la escala del centro de estudios epidemiológicos en depresión (CES-D-IS)}

Está conformada por 4 ítems, con una escala tipo Likert de 0 (ningún día sin el síntoma) hasta 4 ítems (cinco a siete días con el síntoma) que evalúan pensamientos y deseos acerca la muerte (Roberts, 1980). La escala mostró aceptable desempeño psicométrico en Colombia $(\alpha=0,75)$ (CaballeroDomínguez et al., 2020). En la presente investigación, las puntuaciones iguales o superiores a nueve se tomarán como un alto riesgo suicida, similar a un estudio anterior en población colombiana (Campo-Arias et al., 2020). En la presente muestra, el CES-DD-IS mostró un coeficiente de consistencia interna de $\alpha=0,78$. Ejemplo de ítem: He sentido que mi familia estaría mejor si yo estuviera muerto (a).

\subsection{Procedimiento}

Inicialmente, se realizó la presentación de la investigación a los directores de los programas académicos y decanos de las facultades de la Universidad; en segundo lugar, se pidió la autorización con los respectivos docentes de las asignaturas seleccionadas, para acceder a los estudiantes mayores de 18. La recolección de los datos se realizó durante el primer semestre del 2018-I (febrero a mayo) correspondiente al periodo regular de actividades académicas. Se entregó a las dependencias correspondientes un informe con los resultados hallados.

\subsection{Análisis de datos}

Los datos sociodemográficos se analizaron de manera descriptiva, mediante porcentajes y frecuencias. Posteriormente, se dicotomizaron todas las variables estudiadas y se estableció como variable dependiente la salud general percibida (malestar y bienestar) y como independientes las dimensiones de agotamiento (bajo-alto), cinismo (bajo y alto) e ineficacia académica (baja y alta) del síndrome de burnout, e ideación suicida (bajo riesgo y alto riesgo). Para identificar factores asociados a la percepción de la salud general, se realizó un análisis multivariado, mediante regresión logística binaria, se tomaron las variables que mostraron valores de probabilidad menores al $25 \%$ en el análisis bivariado. Al momento de realizar los diferentes modelos 
para salud general percibida, se estimó el cálculo de razón de oportunidad (OR) con IC $95 \%$, y se ajustó con las variables anteriormente señaladas, según las recomendaciones de Greenland (1989) y la prueba de HosmerLemeshow (Hosmer, Taber \& Lemeshow, 1991). Las variables sociodemográficas se incluyeron en el modelo como variables confusoras. Los datos obtenidos fueron procesados mediante el Statistical Package for the Social Sciences (SPSS v25.0).

\subsection{Consideraciones éticas}

El presente estudio fue aprobado por el Comité de Ética en Investigación de una Universidad Pública de Santa Marta, Magdalena, Colombia. La recolección de los datos se llevó a cabo gracias al uso de cuestionarios físicos, previa socialización de los objetivo y justificación de la investigación y firma del consentimiento informado. Los participantes dieron consentimiento voluntario, en congruencia con la Declaración de Helsinki (Asociación Médica Mundial, 2020). Este tipo de investigación no representó riesgo presente o futuro a la salud física o emocional, según la Resolución 8340 de 1993 del Ministerio de Salud de Colombia.

\section{RESULTADOS}

En el presente estudio se contó con la participación de 807 estudiantes universitarios, con edades entre 18 a 47 años $(M=23 ; D E=6,7)$, de los cuales el $24 \%$ son adultos jóvenes y el $60 \%$ son del género femenino. En la Tabla 1 se reportan las características sociodemográficas de los participantes.

Tabla 1.

Variables sociodemográficas de la muestra

\begin{tabular}{cccc}
\hline \multicolumn{2}{c}{ VARIABLES SOCIODEMOGRÁFICAS } & FRECUENCIA & PORCENTAJE \\
\hline \multirow{2}{*}{ Género } & Masculino & 326 & 40 \\
& Femenino & 481 & 60 \\
\multirow{2}{*}{ Edad } & Adultos jóvenes (18-30 años) & 194 & 24 \\
& Adultos (31-47 años) & 613 & 76 \\
\multirow{2}{*}{ Estrato Socioeconómico } & Estrato bajo & 597 & 74 \\
& Estrato medio- alto & 210 & 26 \\
\multirow{2}{*}{ Estado Civil } & Soltero & 446 & 55 \\
& Casado /Unión Libre & 361 & 45 \\
\hline
\end{tabular}

En lo referente a las variables de salud mental se halló que el $28 \%$ de los estudiantes informan malestar general percibido. El $27 \%$ manifiestan alto agotamiento, el $35 \%$ bajo cinismo y el $20 \%$ ineficacia. El $8 \%$ de los estudiantes informan alto riesgo de ideación suicida (Tabla 2). 
Tabla 2.

Variables de Salud Mental

\begin{tabular}{cccc}
\hline & & FRECUENCIA & PORCENTAJE \\
\hline \multirow{2}{*}{ Salud general percibido } & Bienestar & 583 & 72 \\
& Malestar & 224 & 28 \\
\multirow{2}{*}{ Agotamiento } & Baja & 588 & 73 \\
& Alto & 219 & 27 \\
\multirow{3}{*}{ Cinismo } & Bajo & 527 & 65 \\
& Alto & 280 & 35 \\
\multirow{2}{*}{ Ineficacia } & Eficacia & 643 & 80 \\
& Ineficacia & 164 & 20 \\
& Biesgo ideación suicida & 740 & 92 \\
& Bajo riesgo & 67 & 8 \\
\hline
\end{tabular}

En el análisis multivariado, el modelo ajustado para la salud general percibida, sugiere que niveles alto de ideación suicida (OR = 3,16; IC $95 \%$ 1,82 - 5,49), agotamiento alto (OR $=2,22$; IC $95 \% 1,51-3,25)$, cinismo alto $(O R=1,61$; IC $95 \%$ 1,14-2,28) e ineficacia académica (OR = 1,60; IC $95 \% 1,12-2,28$ ) se asociaron a malestar general percibido. Las variables género, edad, estrato socioeconómico, estado civil y tener o no tener hijos no mostraron un comportamiento como variables de confusión para el modelo de salud general percibido (Tabla 3).

Tabla 3.

Factores asociados al malestar acerca de la salud general percibida

\begin{tabular}{ccccccccc}
\hline \multirow{2}{*}{ VARIABLES } & \multicolumn{4}{c}{ MODELO CRUDO } & \multicolumn{4}{c}{ MODELO AJUSTADO } \\
\cline { 2 - 10 } & $\mathbf{P}$ & OR & INFERIOR & SUPERIOR & $\mathbf{P}$ & OR & INFERIOR & SUPERIOR \\
\hline Idea Suicida & 0,00 & 4,80 & 2,85 & 8,06 & 0,00 & 3,16 & 1,82 & 5,49 \\
Agotamiento & 0,00 & 2,98 & 2,08 & 4,27 & 0,00 & 2,22 & 1,51 & 3,25 \\
Cinismo & 0,00 & 2,40 & 1,75 & 3,30 & 0,00 & 1,61 & 1,14 & 2,28 \\
Eficacia académica & 0,00 & 1,93 & 1,38 & 2,69 & 0,00 & 1,60 & 1,12 & 2,28 \\
\hline
\end{tabular}

IC $95 \%$ : intervalo de confianza del $95 \%$; OR: Odds ratio. Hosmer-Lemeshow =3,551; gl = 4; p = 0,470.

\section{DISCUSIÓN}

En la presente investigación se evidenció que el $28 \%$ de estudiante reportan malestar acerca la salud perciba, referido a poco bienestar percibido, a pensamientos y sentimientos negativos acerca de sí mismo y funcionamiento como insatisfactorio tanto a nivel físico, psíquico y social. En investigaciones en contextos internacionales, se encontró una prevalencia cercana a la reportada en el presente estudio. González \& Palacio (2017), en una muestra de 313 estudiantes de odontología, informó que $26 \%$ de los estudiantes universitarios de Cali perciben malestar general. En otro estudio se observó, en 143 estudiantes, que el $19 \%$ de los universitarios de La Habana perciben 
su salud como mala (Pacheco, Michelena, Mora \& Miranda, 2014); y en 300 estudiantes de psicología y medicina de Quito se identificó que el 4,3 \% tienen una percepción de salud medio baja (Álvarez \& Reyes, 2019). Los hallazgos anteriores señalan que los estudiantes universitarios en su proceso formativo perciben malestar acerca de su salud general, y podría tener un efecto negativo en el rendimiento académico y en el bienestar en las diferentes áreas de funcionamiento (Díaz, Martínez \& Zapata, 2017; Hernández, Álvarez \& Ruiz, 2019).

El presente estudio encontró asociación entre ideación suicida a malestar general percibido. Estos resultados son coherentes con referentes internacionales. En una investigación adelantada con estudiantes de medicina noruegos se halló relación entre la ideación suicida y el malestar general percibido definido como angustia mental (Tyssen, Vaglum, Grønveld \& Ekeberg, 2001). Eguiluz \& Mera (2014) informan asociación entre baja percepción de bienestar asociado a riesgo de sufrir depresión o riesgo suicida. Coherentemente con los anteriores hallazgos, en adolescentes mexicanos se encontró que la percepción del malestar físico y psicológico se asoció de manera significativa y positiva con la presencia de ideación suicida (Domínguez, Vargas, Castro, Medina \& Huertas, 2019).

El deseo, el pensamiento o bien la cognición orientada a imágenes sobre la pérdida del sentido de la vida o autodestructivas (ideación suicida) es un factor de riesgo para salud general percibida de los estudiantes. La visión negativa frente a la vida genera una situación conflictiva acompañada de tensión emocional o/y física, impulsividad y agresión, por lo tanto, problemas internalizados y externalizados, que predispone el malestar general percibido. Estos resultados son coherentes con los reportados por diversos estudios (Guarino, Gavidia, Antor \& Caballero, 2000; Calvo, Sánchez \& Tejeda, 2003; Hernández \& Gómez, 2006; Cova, Alvial, Aro, Bonifettu, Hernández \& Rodríguez, 2007; Patiño, Arboleda, Arango, Botero \& Arango, 2019). Lo anterior puede explicarse debido a la mediación de los pensamientos de optimismo y de esperanza relacionada con la vida y con los significados que el sujeto atribuye a su salud general percibido. Los pensamientos optimistas son modulados por constructos cognitivos-afectivos que favorece la construcción mental de creencias y de valores que promueven el sentirse bien consigo mismo y con los demás, el autocuidado, y recursos psicológicos para la construcción de experiencias y escenarios positivos en los diferentes entornos, especialmente en el académico. Cuando un individuo es optimista muestra una tendencia a explicar los sucesos adversos como causas de atribuciones externas y se 
interesan en mayor medida en esforzarse para cumplir sus metas (Torija, Sierra \& Sandoval, 2017).

En el presente estudio cada una de las dimensiones del síndrome (agotamiento, el cinismo e ineficacia académica) se asoció a malestar acerca la salud general percibida. Es importante señalar, que las dimensiones de agotamiento y cinismo, consideradas corazón del burnout, y la ineficacia académica son un factor de riesgo para la salud general percibida. Estos resultados coinciden con los hallazgos de Saravia (2013) y Hernández, Marques, Jiménez \& Rodríguez (2016) quienes muestran asociación del agotamiento y cinismo a salud general percibida. Consistente con los precedentes anteriores, el burnout como constructo unidimensional se asoció negativamente con salud general en estudiantes de medicina chinos (Fan, Kosik, Huang, Gjiang, Lien, Zhao et al., 2017).

Los sentimientos y pensamientos, desinterés, autosabotaje y poca competencia frente a las demandas académicas se asocian negativamente con el agotamiento (Caballero, Bresó \& González, 2015). Es decir, la percepción de ineficacia o incompetencia personal caracterizada por los procesos de agenciamiento cognitivo, motivacional limita los recursos para hacer frente al agotamiento (Villa, Zuluaga \& Restrepo, 2013). Desde esta perspectiva, los niveles de estrés crónico, insidioso y negativo, mediado por la ineficacia académica, podría explicar las dificultades del estudiante para trabajar productivamente y valorar su proceso de aprendizaje como satisfactorio (Sreeramareddy, Shankar, Binu, Mukhopadhyay, Ray \& Menezes, 2007; Vélez \& Roa, 2005); en consecuencia, el estudiante tendría menos recursos psicológicos para realizar un afrontamiento que le permita alcanzar un adecuado desempeño académico (Vaez, Kristenson \& Laflamme, 2004) y evitar la deserción académica (Borquéz, 2005; Caballero, Gallo y Suárez, 2018).

\section{CONCLUSIONES}

Se concluye que la ideación suicida y las dimensiones del síndrome de burnout, agotamiento, el cinismo y la ineficacia son factores de riesgo a la salud general percibida en los estudiantes universitarios.

Este estudio aporta a la comprensión de los estresores crónicos y de la ideación suicida en el malestar general percibido. La identificación oportuna de dichos factores puede ayudar a prevenir comportamiento de riesgo para la salud y el bienestar de los estudiantes, mediante programas de prevención adaptados culturalmente a los contextos sociales y que resalten el papel de 
la autoeficacia académica, para organizar y ejecutar tareas académicas de manera eficaz, considerada factor protector para la salud general (Bandura, 1997; Liébana, 2012; Schönfeld, Brailovskaia, Bieda, Chi Zhang, \& Margraf, 2016).

Se recomienda realizar investigaciones que consideren otras variables como la depresión, ansiedad, obesidad y estilos de vida asociadas a la salud general percibida en estudiantes de básica secundaria, media y universitarios que permitan crear un insumo para programas de intervención que fomenten acciones psicoeducativas basadas en la educación de la salud, que promueva la regulación de las emociones, y la prospectiva de vida, son necesarios para prevenir estrés y la ideación suicida, y el subsecuente desarrollo del comportamiento de suicidio. Este estudio también aporta referentes empíricos a las dependencias de Bienestar Universitarios de las Instituciones de Educación Superior para el desarrollo y formulación de lineamientos de políticas encaminadas al bienestar de los estudiantes universitarios.

Además, se sugiere incluir estudiantes de programas académicos de diversa naturaleza y variables sociodemográficas y culturales, para que los datos que arrojen se puedan extrapolar con mayor fiabilidad. Es importante tener en cuenta que la presente investigación tiene sus limitantes, su diseño transversal no permite establecer la dirección de causalidad entre las variables estudiadas, sugiriendo un diseño de tipo longitudinal que pueda mostrar una causa-efecto, haciendo la investigación más enriquecedora.

Conflicto de interés: Los autores declaran que no existe, de manera directa o indirecta, ningún tipo de conflicto de intereses, financieros, académicos ni personales para la publicación de este artículo de investigación.

Agradecimiento: A la Universidad del Magdalena por todo el apoyo administrativo y académico para la realización de la presente investigación.

Financiamiento: Este trabajo fue financiado por Convocatoria para la financiación de proyectos de investigación, desarrollo experimental, innovación y creación artística y cultural - Fonciencias del 2017, de la Universidad del Magdalena, Santa Marta, Colombia.

\section{REFERENCIAS}

Aburto, C., Díaz, K., \& López, P. (2017). Ideación suicida en adolescentes del área rural: estilo de crianza y bienestar psicológico. Revista Colombiana de Enfermería, 15(12), 50-61. https://doi.org/10.18270/rce.v15i12.2136 
Álvarez, D. \& Reyes, F. (2019). Salud mental percibida y distrés psicológico en estudiantes de Ciencias Psicológicas y Médicas de la Universidad Central del Ecuador (Tesis de maestría). Universidad Central del Ecuador, Quito, Ecuador. http://www.dspace. uce.edu.ec/bitstream/25000/18275/1/T-UCE-0007-CPS-106.pdf

Aradilla, A., \& Tomás, J. (2011). The role of emotional intelligence in nursing. (1a Ed). New York, NY: Nova Sciences Publishers.

Aranceli, S., Perea, P., \& Ormeño, R. (2006). Evaluación de niveles, situaciones generadoras y manifestaciones de estrés académico en alumnos de tercer y cuarto año de una Facultad de Estomatología. Revista Estomatológica Herediana, 16(1), 15-20. http://www.redalyc.org/articulo.oa?id=421539345004

Arango, S., Castaño, J., Henao, C., Jiménez, D., López, A., \& Páez, M. (2010). Síndrome de burnout y factores asociados en estudiantes de I a X semestre de la Facultad de Medicina de la Universidad de Manizales (Colombia), 2009. Archivo de Medicina, 10(2), 110-126. https://doi.org/10.30554/archmed.10.2.1291.2010

Ardila, R. (2003). Calidad de vida: una definición integradora. Revista Latinoamericana de Psicología, 35(2), 161-164. http://www.redalyc.org/articulo.oa?id=80535203

Asociación Médica Mundial (2020). Declaración de Helsinki. Principios éticos para las investigaciones médicas en seres humanos. https://www.wma.net/es/ policies-post/declaracion-de-helsinki-de-la-amm-principios-eticos-para-las-investigaciones-medicas-en-seres-humanos/.

Balsera, M., Chavasco, G., López, R., Pérez, A., Sánchez, M., \& Villena, C. (2016). Estudio del Burnout en estudiantes de la Universidad de Granada. Influencia moduladora de variables de inteligencia emocional y clasificación por cursos. ReiDoCrea, 5(2), 27-32. https://www.ugr.es/ reidocrea/5-2-5.pdf

Bandura, A. (1997). Self-efficacy: The exercise of control. Editorial: Macmillan.

Becerra, S. (2013). Rol del estrés percibido y su afrontamiento en las conductas de salud de estudiantes universitarios de Lima (Tesis de maestría). Pontificia Universidad Católica del Perú, Lima. http://tesis.pucp.edu.pe/repositorio/ handle/20.500.12404/5013

Borquéz, S. (2005). Burnout, o estrés circular en profesores. Pharos, 1, 23-34. https:// www.redalyc.org/pdf/208/20811103.pdf

Caballero, C., Bresó, E., \& González, O. (2015). Burnout en estudiantes universitarios. Psicología desde el Caribe, 32(3), 424-441. 15. http://dx.doi.org/10.14482/ psdc.32.3.6217

Caballero, C., Gallo, Y., \& Suárez, Y. (2018). Algunas variables de salud mental asociadas con la propensión al abandono de los estudios universitarios. Psychologia, 12(2), 37-46. https://doi.org/10.21500/19002386.3466

Caballero-Domínguez, C. (2012). El burnout académico: Prevalencia y factores asociados en estudiantes universitarios del área de la salud de la ciudad de Barranquilla (Doctoral dissertation). Universidad del Norte, Barranquilla, Colombia.

Caballero-Domínguez, C. C, Jiménez-Villamizar, M. P, \& Campo-Arias, A. (2020). Suicide risk during the lockdown due to coronavirus disease (COVID-19) in Colombia. Death Studies, 44, 1-6. https://doi.org/10.1080/07481187.2020.1784312

Caballero-Domínguez, C., Abello, R., \& Palacio, J. (2007). Relación del burnout y el rendimiento académico con la satisfacción frente a los estudios en estudiantes universitarios. Avances en psicología latinoamericana, 25(2), 98-111. https:// revistas.urosario.edu.co/index.php/apl/article/viewFile/1208/1076

Calvo, J. M., Sánchez, R., \& Tejada, P. A. (2003). Prevalencia y factores asociados a ideación suicida en estudiantes universitarios. Revista de Salud Pública, 5(2), 123-143. http://www.scielo.org.co/pdf/rsap/v5n2/v5n2a02.pdf 
Campo-Arias, A., Suárez-Colorado, Y. P., \& Caballero-Domínguez, C. C. (2020). Asociación entre consumo de cannabis y riesgo de suicidio en adolescentes escolarizados de Santa Marta, Colombia. Biomédica, 40(3). https://revistabiomedica.org/index.php/ biomedica/article/view/4988

Carlin, M., \& Garcés de los Fayos Ruiz, E. (2010). El síndrome de Burnout: evolución histórica desde el contexto laboral al ámbito deportivo. Anales de Psicología, 26(1), 169-180. https://www.redalyc.org/articulo.oa?id=167/16713758020

Ceballos, G., \& Suárez, Y. (2012). El suicidio. (1a ed). Santa Marta: Editorial Unimagdalena.

Chau, C., \& Saravia, J. C. (2014). Adaptación universitaria y su relación con la salud percibida en una muestra de jóvenes de Perú. Revista Colombiana de Psicología, 23(2), 269-284. http://dx.doi.org/10.15446/rcp.v23n2.41106

Chau, C., \& Tavera, M. (2012). Informe proyecto PUCP Saludable. I. Diagnóstico situacional. Manuscrito inédito. Pontificia Universidad Católica del Perú, Lima, Perú.

Consorcio de Universidades. (2006). Perfil de los estudiantes del Consorcio de Universidades: resumen y análisis. Lima: Consorcio de Universidades. http://www.scielo.org.pe/scielo.php?script=sci_nlinks\&ref=1485227\&pi$\mathrm{d}=$ S0254-9247201600020000200017\&lng=es

Cova, F., Alvial, W., Aro, M., Bonifetti, A., Hernández, M., \& Rodríguez, C. (2007). Problemas de salud mental en estudiantes de la Universidad de Concepción. Terapia psicológica, 25(2), 105-112. http://dx.doi.org/10.4067/S0718-48082007000200001

Dasgupta, S., \& Hazra, S. (2011). Does student's emotional intelligence play role in their suicidal ideation. Indian Journal of Community Psychology, 7(1), 190-197. https:// www.researchgate.net/publication/260057285_Does_Student's_Emotional_ Intelligence_Play_Role_in_their_Suicidal_Ideation

Díaz, S., Martínez, M., \& Zapata, A. (2017). Rendimiento académico y calidad de vida relacionada con la salud en estudiantes de odontología. Salud Uninorte, 33(2), 86-91. http://dx.doi.org/10.14482/sun.33.2.10540

Domínguez, R., Vargas, E., Castro, R., Medina, R., \& Huertas, C. (2019). Ciberacoso como factor asociado al malestar psicológico e ideación suicida en adolescentes escolarizados mexicanos. Acta Universitaria, 29, 1-10. http://doi.org/10.15174/ au.2019.2295

Eguiluz R., \& Mera M. (2014). Medición del riesgo suicida, bienestar psicológico y estructura familiar en estudiantes de primer ingreso a las carreras de la salud. Revista de Investigación en Psicología, 17(1), 43-53. https://doi.org/10.15381/ rinvp.v17i1.8971

Espinás, J., Riba, M., Borrás, J., \& Sánchez, V. (1995). Estado de salud, utilización de servicios sanitarios y morbilidad declarada: Aplicación del análisis de correspondencia. Revista Española de Salud Pública, 69(2), 195-205. https://www. mscbs.gob.es/biblioPublic/publicaciones/recursos_propios/resp/revista_cdrom/ VOL69/69_2_195.pdf

Fan, A., Kosik, R., Huang, L., Gjiang, Y., Lien, S., Zhao, X. et al. (2017). Burnout in Chinese medical students and residents: an exploratory cross-sectional study. The Lancet, 390, S84. https://doi.org/10.1016/S0140-6736(17)33222-1

Flores, M., Chávez, M., \& Aragón, L. (2016). Situaciones que generan ansiedad en estudiantes de Odontología. Journal of Behavior, Health \& Social Issues, 8(2) 35-41. https://doi.org/10.1016/j.jbhsi.2016.11.004

González, A., \& Palacios, J. (2017). Estilo de vida y salud percibida en estudiantes de un programa de odontología de una universidad de Santiago de Cali (Tesis Maestría). Pontificia Universidad Javeriana, Cali, Colombia. http://vitela.javerianacali.edu.co/ bitstream/handle/11522/7984/Estilos_vida_salud.pdf?sequence=1\&isAllowed=y 
Gracia, E., Herrero, J., \& Musitu, G. (1996). Salud y comunidad: Evaluación de los recursos y estresores. Madrid, España: Editorial Síntesis S.A.

Greenland, S. (1989). Modeling and variable selection in epidemiologic analysis. American Public Health Association, 79(3), 340-349. https://doi.org/10.2105/ ajph.79.3.340

Guarino, L., Gavidia, I., Antor, M., \& Caballero, H. (2000). Estrés, salud mental y cambios inmunológicos en estudiantes universitarios. Psicología Conductual, 8(1), 57-71. https://dialnet.unirioja.es/servlet/articulo?codigo $=2827040$

Hanna, M., Ocampo, M., Janna, N., Mena, M., y Torreglosa, L. (2020). Redes sociales y calidad de vida relacionada con la salud en estudiantes universitarios. Revista Cuidarte, 11(1), 1-13. http://dx.doi.org/10.15649/cuidarte.953

Hederich-Martínez, C., \& Caballero-Domínguez, C. (2016). Validación del cuestionario Maslach Burnout Inventory-Student Survey (MBI-SS) en contexto académico colombiano. Revista CES Psicología, 9(1), 1-15. http://www.scielo.org.co/scielo. php?script=sci_arttext\&pid=S2011-30802016000100002

Herazo, Y., Núñez, N., Sánchez, L., Vásquez, F., Lozano, A., Torres, E., \& Valdelamar, A. (2020). Estilos de vida relacionados con la salud en estudiantes universitarios. Retos: nuevas tendencias en educación física, deporte y recreación, 38, 547-551. https://recyt.fecyt.es/index.php/retos/article/view/72871

Hernández, F., Marques, A., Jiménez, J., \& Rodríguez, B. (2016). El síndrome de burnout y la salud mental de los estudiantes de grado en enfermería. Revista Portuguesa de Enfermagem de Saúde Mental (3), 79-84. http://dx.doi.org/10.19131/rpesm.0122

Hernández, Q., \& Gómez, E. (2006). Evaluación del riesgo suicida y estrés asociado en adolescentes estudiantes mexicanos. Revista Mexicana de Psicología, 23(1), 45-52. https://www.redalyc.org/articulo.oa?id=243020646006

Hernández-Ávila, M., Garrido-Latorre, F., \& López, S. (2000). Diseño de estudios epidemiológicos. Salud Pública de México, 42(2), 144-154. https://www.scielosp.org/ article/spm/2000.v42n2/144-154/

Hernández-García, E., Álvarez-Martínez, B., \& Ruiz-Ruisánchez, A. (2019). Salud y desempeño en estudiantes de distintos niveles académicos. Revista del Hospital Universitario Juárez de México, 86(4), 196-201. https://www.medigraphic.com/ cgi-bin/new/resumen.cgi?IDARTICULO=90747\#

Hosmer, D., Taber, S., \& Lemeshow, S. (1991) The importance of assessing the fit of logistic regression models: a case study. American Public Health Association, 81(12), 1630-1635. https://doi.org/10.2105/ajph.81.12.1630

Kumar, S., Dixit, V., Chaudhury, S., \& Kenswar, D. K. (2013). Racial differences in suicidal ideation among school going adolescents. Industrial psychiatry journal, 22(2), 118. https://doi.org/10.4103/0972-6748.132924

Liébana-Presa, C. (2012). Salud emocional en estudiantes universitarios de enfermería y fisioterapia de Castilla y León (Tesis doctoral). Universidad de Córdoba, España. https://helvia.uco.es/xmlui/handle/10396/8088

Marenco, A., Suárez, Y., \& Palacio, J. (2017). Burnout académico y síntomas Relacionados con problemas de salud mental en universitarios colombianos. Psychologia, 11(2), 45-55. https://doi.org/10.21500/19002386.2926

Martos, Á., Pérez-Fuentes, M., Del Mar, M., Gázquez, J., Del Mar, M., \& Barragán, A. (2018). Burnout y engagement en estudiantes de Ciencias de la Salud. European Journal of Investigation in Health, Psychology and Education, 8(1), 23-36. https:// doi.org/10.30552/ejihpe.v8i1.223

Muñoz, J., Pinto, V., Callata, H., Napa, N., \& Perales, A. (2006). Ideación suicida y cohesión familiar en estudiantes preuniversitarios entre 15 y 24 años, Lima 2005. 
Revista Peruana de Medicina Experimental y Salud Pública, 23(4), 239-246. https:// www.redalyc.org/articulo.oa?id=36323402

Olarte, M. (2018). Salud y enfermedad, dos construcciones culturales. Revista Biosalud, 17(1), 67-79. https://doi.org/10.17151/biosa.2018.17.1.7

Organización Mundial de la Salud - OMS. (2001). Informe sobre la Salud en el Mundo. http://www.who.int/whr/2001/en/whr01_es.pdf?ua=1

Organización Mundial de la Salud. (2004). La organización del trabajo y el estrés: estrategias sistémicas de la solución de problemas para empleados, personal directivo y representantes sindicales. https://www.who.int/occupational_health/publications/pwh3sp.pdf

Organización Mundial de la Salud. (2006). Constitución de la Organización Mundial de la Salud. Documentos básicos, suplemento de la 45a edición. https://www.who.int/ governance/eb/who_constitution_en.pdf?ua=1

Pacheco, M., Michelena, M., Mora, R., \& Miranda, O. (2014). Calidad de vida relacionada con la salud en estudiantes universitarios. Revista Cubana de Medicina Militar, 43(2), 157-168. http://scielo.sld.cu/scielo.php?script=sci_arttext\&pi$d=S 0138-65572014000200004 \&$ Ing=es\&tlng=pt.

Palacio, J., Caballero-Domínguez, C., González, O., Gravini, M., \& Contreras, K. (2012). Relationship between burnout and coping strategies with GPA in university students. Universitas Psychologica, 11(2), 535-544. http://www.scielo.org.co/ scielo.php?script=sci_arttext\&pid=S1657-92672012000200015

Patiño-Villada, F. A.; Arboleda-Serna, V. H; Arango-Vélez, E. F; Botero-Restrepo, S. \& Arango-Paternina, C. (2019). Depresión, ansiedad y calidad de vida relacionada con la salud, en estudiantes universitarios de educación física y deportes. Educación Física y Deporte, 38(2). http://doi.org/10.17533/udea.efyd.v38n2a02

Roberts, R. (1980). Reliability of the CES-D Scale in different ethnic contexts. Psychiatry Research, 2(2), 125-134. https://doi.org/10.1016/0165-1781(80)90069-4

Rosales, Y., \& Rosales-Paneque, F. (2013). Burnout estudiantil universitario. Conceptualización y estudio. Revista Salud Mental, 36(4), 337-345. http://www.scielo.org.mx/ $\mathrm{pdf} / \mathrm{sm} / \mathrm{v36n} 4 / \mathrm{v} 36 \mathrm{n} 4 \mathrm{a} 9 . \mathrm{pdf}$

Salanova, M., Martínez, I., Bresó, E., Llorens, S., \& Grau, R. (2005). Bienestar psicológico en estudiantes universitarios: facilitadores y obstaculizadores del desempeño académico. Anales de Psicología, 21(1), 170-180. https://dialnet.unirioja.es/ servlet/articulo?codigo $=1214651$

Saravia, J. C. (2013). Factores psicológicos y conductuales de la salud en un grupo de universitarios de Lima Metropolitana (Tesis de licenciatura). Pontificia Universidad Católica del Perú, Lima, Perú. http://tesis.pucp.edu.pe/repositorio/bitstream/ handle/123456789/5009/SARAVIA_DRAGO_JUAN_CARLOS_FACTORES.pdf?sequence $=1 \&$ is Allowed $=y$

Schaufeli, W. B., Salanova, M., González-Romá, V., \& Bakker, A. (2002). The measurement of burnout and engagement: A confirmatory factor analytic approach. Journal of Happiness Studies, 3, 71-92. https://www.wilmarschaufeli.nl/publications/ Schaufeli/178.pdf

Schönfeld, P., Brailovskaia, J., Bieda, A., Chi Zhang, X. C., \& Margraf, J. (2016). The effects of daily stress on positive and negative mental health: Mediation through self-efficacy. International Journal of Clinical and Health Psychology, 16(1), 1-10. https:// doi.org/10.1016/j.ijchp.2015.08.005

Schwartzmann, L. (2003). Calidad de vida relacionada con la salud: aspectos conceptuales. Ciencia y Enfermería, 9(2), 9-21. https://doi.org/10.4067/S071795532003000200002 
Seligman, M. E., \& Peterson, C. (2003). Positive clinical psychology. En L.G. Aspinwall \& U.M. Staudinger (Eds.). A Psychology of human strengths: Fundamental questions and future directions for a positive psychology (pp.305-317). Washington, D.C: American Psychological Association.

Sreeramareddy, C., Shankar, P., Binu, S., Mukhopadhyay, C., Ray, B., \& Menezes, R. G. (2007). Psychological morbidity, sources of stress and coping strategies among undergraduate medical students of Nepal. BMC medical education, 7, 26. https:// doi.org/10.1186/1472-6920-7-26

Torija, C., Sierra, V., \& Sandoval, A. (2017). Bienestar psicológico, conductas asociadas al suicidio y el desempeño académico en estudiantes universitarios. Revista Electrónica de Psicología Iztacala, 20(3), 11-24. http://www.revistas.unam.mx/ index.php/repi/article/view/61757/54382

Tyssen, R., Vaglum, P., Grønveld, N. T., \& Ekeberg, $\emptyset$. (2001). Suicidal ideation among medical students and young physicians: a nationwide and prospective study of prevalence and predictors. Journal of Affective Disorders, 64(1), 69-79. https://doi. org/10.1016/s0165-0327(00)00205-6

Vaez, M., Kristenson, M., \& Laflamme, L. (2004). Perceived quality of life and self-rated health among first-year university students. Social Indicators Research, 68(2), 221-234. https://www.jstor.org/stable/27522115

Vélez, A., \& Roa, C. (2005). Factores asociados al rendimiento académico en estudiantes de medicina. Educación Médica, 8(2), 74-82. http://scielo.isciii.es/pdf/edu/v8n2/ original1.pdf

Victoria, C., \& González, I. (2000). La categoría Bienestar psicológico. Su relación con otras categorías sociales. Revista Cubana de Medicina General Integral, 6(16), 586-592. http://scielo.sld.cu/scielo.php?script=sci_arttext\&pid=S0864-21252000000600010

Villa, I., Zuluaga, C., \& Restrepo, L. (2013). Propiedades psicométricas del Cuestionario de Salud General de Goldberg GHQ-12 en una institución hospitalaria de la ciudad de Medellín. Avances en Psicología Latinoamericana, 31(3), 532-45. http://www. scielo.org.co/pdf/apl/v31n3/v31n3a07.pdf

Viñas, F., \& Chaparrós, C. (2000). Afrontamiento del periodo de examen y sintomatología somática autoinformada en un grupo de estudiantes universitarios. Revista Psicología.com, 4(1). https://psiquiatria.com/bibliopsiquis/afrontamiento-del-periodo-de-examenes-y-sintomatologia-somatica-autoinformada-en-un-grupo-de-estudiantes-universitarios/

Wilcox, H. C., Arria, A. M., Caldeira, K. M., Vincent, K. B., Pinchevsky, G. M., \& O’Grady, K. E. (2010). Prevalence and predictors of persistent suicide ideation, plans, and attempts during college. Journal of affective disorders, 127(1), 287-294. https://doi. org/10.1016/j.jad.2010.04.017

Esta obra está bajo: Creative commons attribution 4.0 international license. El beneficiario de la licencia tiene el derecho de copiar distribuir, exhibir y representar la obra y hacer obras derivadas siempre y cuando reconozca y cite la obra de la forma especificada por el autor o el licenciante.

\section{(cc) BY}

